\title{
Qualidade de vida e espiritualidade dos cuidadores informais de pacientes oncológicos: uma revisão narrativa
}

\author{
Quality of life and spirituality of informal caregivers of cancer patients: a narrative review \\ Calidad de vida y espiritualidad de cuidadores informales de pacientes oncológicos: una \\ revisión narrativa
}

Andressa Carlos De Oliveira" ${ }^{1 *}$, Julyana Krüger Pilon ${ }^{1}$, Quésia Silva Dos Santos ${ }^{1}$, Danilo Anderson Pereira ${ }^{2}$, Andreza Carcará Rocha ${ }^{3}$, Gabriela Alves Luz ${ }^{4}$, Ives do Nascimento Monteiro ${ }^{4}$, Eise Souza do Vale ${ }^{5}$, Ana Clara Santos Teixeira ${ }^{6}$, Ítalo Filipe Cardoso Amorim¹.

\begin{abstract}
RESUMO
Objetivo: Analisar as condições que interferem na qualidade de vida dos cuidadores de pacientes oncológicos e a influência da espiritualidade nesse contexto. Revisão bibliográfica: Ao desempenhar o cuidado, 0 indivíduo se disponibiliza a atuar além da demanda física do paciente, de maneira a acolher e entender as particularidades, limitações e necessidades do outro, fornecendo inclusive, carinho, atenção e empatia. A sobrecarga de cada cuidador pode variar, de acordo com o estágio da doença ou com os aspectos ligados ao estado do paciente. As práticas diárias espirituais, auxiliam os cuidadores a enfrentar e aceitar a doença do familiar doente, além de permitir também uma postura mais positiva por parte do doente, ajudando na regulação de emoções negativas. A manutenção da saúde do cuidador informal é essencial para a prática saudável do ofício. Quando ocorre o reconhecimento dos fatores que levam a vulnerabilidades e proteção do cuidador informal principal do paciente com câncer, há a possibilidade de melhora da qualidade de vida do mesmo. Considerações finais: Ao ressaltar o cuidador como elemento que necessita de atenção, podemos prevenir o seu adoecimento e garantir a continuidade da realização dos cuidados ao familiar dependente.
\end{abstract}

Palavras-chave: Qualidade de vida, Espiritualidade, Oncologia.

\begin{abstract}
Objective: To analyze the conditions that interfere in the quality of life of caregivers of cancer patients and the influence of spirituality in this context. Bibliographic review: When performing care, the individual is available to act beyond the physical demand of the patient, in order to welcome and understand the particularities, limitations and needs of the other, including providing affection, attention and empathy. The burden of each caregiver may vary, according to the stage of the disease or aspects related to the patient's condition. Daily spiritual practices help caregivers to face and accept the illness of the sick family member, in addition to allowing a more positive attitude on the part of the patient, helping to regulate negative emotions. Maintaining the health of the informal caregiver is essential for the healthy practice of the profession. When the factors that lead to vulnerabilities and protection of the main informal caregiver of cancer patients are recognized, there is the possibility of improving their quality of life. Final considerations: By highlighting the caregiver as an element that needs attention, we can prevent their illness and ensure the continuity of care for the dependent family member.
\end{abstract}

Keywords: Quality of life, Spirituality, Medical oncology.

\footnotetext{
${ }^{1}$ Faculdade Santo Agostinho (FASA), Vitória da Conquista - BA. *E-mail: dressaoliv.7@gmail.com

2 Universidade Nove de Julho (UNINOVE), Bauru - SP.

${ }^{3}$ Centro Universitário UniFacid, Teresina - PI.

${ }^{4}$ Universidade CEUMA, São Luis - MA.

5 Universidade Positivo, Curitiba - PR.

${ }^{6}$ Faculdade Santo Agostinho de Itabuna (FASAI), Itabuna - BA.
} 


\section{RESUMEN}

Objetivo: Analizar las condiciones que interfieren en la calidad de vida de los cuidadores de pacientes oncológicos y la influencia de la espiritualidad en ese contexto. Revisión bibliográfica: Al realizar el cuidado, el individuo está disponible para actuar más allá de la demanda física del paciente, con el fin de acoger y comprender las particularidades, limitaciones y necesidades del otro, incluso brindando afecto, atención y empatía. La carga de cada cuidador puede variar, según el estadio de la enfermedad o aspectos relacionados con el estado del paciente. Las prácticas espirituales diarias ayudan a los cuidadores a enfrentar y aceptar la enfermedad del familiar enfermo, además de permitir una actitud más positiva por parte del paciente, ayudando a regular las emociones negativas. Mantener la salud del cuidador informal es fundamental para el ejercicio saludable de la profesión. Cuando se reconocen los factores que conducen a la vulnerabilidad y protección del principal cuidador informal de pacientes con cáncer, existe la posibilidad de mejorar su calidad de vida. Consideraciones finales: Al destacar al cuidador como un elemento que necesita atención, podemos prevenir su enfermedad y asegurar la continuidad del cuidado del familiar dependiente.

Palabras clave: Calidad de vida, Espiritualidad, Oncología médica.

\section{INTRODUÇÃO}

O câncer é formado pelo descontrole da divisão celular, resultando no crescimento desordenado de células que tendem a invadir tecidos e órgãos, podendo progredir para outras regiões do corpo e acarretar transtornos funcionais. $O$ número de pessoas com algum tipo de câncer vem aumentando significativamente em todo 0 mundo, gerando mudanças nos perfis sociodemográficos da população mundial, tornando-se a maior causa de morbidade e mortalidade nas últimas décadas (TAVARES DS, et al., 2020).

Essa enfermidade se encaixa nas doenças não transmissíveis, de caráter crônico e na grande parte das vezes, incapacitante, que requer cuidados contínuos tanto em meio hospitalar quanto domiciliar, devido aos elevados índices de sequelas e limitações geradas pela mesma. Apresentando repercussões de ordem epidemiológica, econômica e social (COPPETTI CL, et al., 2020).

Cada vez mais, o sistema de cuidado tem incentivado o ambiente domiciliar como local de atenção ao paciente oncológico. Essa realocação do atendimento, faz com que as famílias sejam responsabilizadas e solicitadas para realizar o papel de prestadora de cuidados, ainda que muitas vezes, não preparadas para assumir o doente. A atenção domiciliar surge como proposta para auxiliar a população fora do meio hospitalar, servindo as necessidades dos habitantes, além de ser uma estratégia alternativa para a redução de custos e tempo de internação (FERREIRA MLSM, et al., 2018).

Uma das classificações utilizadas para os cuidadores que prestam assistência ao enfermo, envolve o tipo de vínculo estabelecido. Nomeando cuidador informal, à pessoa que se responsabiliza pelo cuidado, atuando, acompanhando e supervisionando o indivíduo debilitado, podendo ser um familiar, vizinho, amigo ou membro da comunidade. E considera cuidador formal, os profissionais, como os da área de saúde e instituições que se encarregam do atendimento a partir de um vínculo empregatício (TAVARES DS, et al., 2020).

Ainda, entre os cuidadores, existem aqueles que exercem o papel principal e aqueles que exercem o papel secundário no auxílio. O primeiro tipo, assume a maior parte ou toda a responsabilidade, se encarregando da maioria das tarefas do cuidar. Em contrapartida, o segundo tipo complementa a atenção, são os membros da comunidade, familiares, amigos, profissionais que geralmente dão menor suporte (SANTANA ACO, et al., 2021).

O cuidador informal tem o papel de cuidar de alguém que careça de acompanhamento, por possuir alguma deficiência ou doença, não sendo requisito apresentar formação técnica para realizar tais tarefas, como limpeza, locomoção, alimentação e medicação de rotina, correspondendo a um sujeito que atua no auxílio temporário ou permanente a outra pessoa que esteja necessitando de apoio (SILVA BCA, et al., 2019).

Estes cuidadores, ajudam os pacientes no processo do tratamento, buscando zelar pelo bem-estar do outro. Sendo de sua ocupação: resolver questões burocráticas, manutenção, transporte, apoio emocional, 
social e assistência financeira, além de assistência física para trabalhos domésticos. Além disso, a família é o principal núcleo de origem do cuidador e as mulheres adultas e idosas se destacam nessa posição. Porém, há também casos em que o cuidado é fornecido por homens, crianças ou adolescentes. Algumas situações podem influenciar esta decisão, como a proximidade parental (esposas e filhas), afetiva ou física e o gênero feminino (ABREU AISCS e JÚNIOR ALC, 2018).

O adoecimento de um ente ocasiona mudanças organizacionais da família, promovendo um reajuste de seus papéis, surgindo nesse contexto, a figura do cuidador que normalmente está inserido no próprio meio pessoal. Por exemplo, a mãe, cuidadora da família, ao adoecer, passa a ser cuidada pelos seus filhos, o pai passa a ser cuidado pela esposa ou filhos. Cada um, apropria-se dessa nova função, naturalmente, sem questionamentos sobre o desejo, disponibilidade e a vontade do cuidador, não ocorrendo muitas vezes um acordo explícito de quem vai desempenhar essa função (VALE JMM, et al., 2019).

O cuidador, frequentemente, torna-se encarregado de múltiplas funções, muitas vezes desconhecidas pela maior parte deles, além de lidar com mudanças no quadro clínico do paciente e a possibilidade de óbito. Essa realidade revela que o cuidado no domicílio é complexo, principalmente na fase avançada da enfermidade. Logo, essas múltiplas obrigações podem gerar efeitos na vida do cuidador, que se manifestam através de sinais e sintomas físicos e psíquicos, além dos desgastes sociais e econômicos que produzem alterações na qualidade da sua vida (OLIVEIRA MBP, et al., 2017).

A qualidade de vida é descrita sobre um aspecto subjetivo, de percepção pessoal e multidimensional que envolve múltiplos domínios, dos quais incluem comumente as características sociais, saúde física, estado psicológico, condição econômica e padrão espiritual. Sendo assim, os cuidadores de doentes oncológicos podem ter uma qualidade de vida variável, a depender da esfera clínica do familiar, da perspectiva do cuidador e do meio onde se insere (MIGUEL I, et al., 2019).

Diante disso, surge um elemento relevante que influencia o processo de saúde. Trata-se da espiritualidade e das práticas religiosas, vistas como recurso para o enfrentamento dos problemas diários, eventos estressantes e traumáticos, como aqueles decorrentes do processo de adoecimento e hospitalização (VIANNA MLGS e SOUZA W, 2017).

Grande parte dos cuidadores procuram um motivo ou propósito positivo frente a situações difíceis, contando com crenças e artifícios espirituais para lidar com as mudanças e transformações impostas pela enfermidade, para alcançar uma melhor qualidade de vida para si próprio e para seu familiar adoecido. Ao realizar essas práticas, as pessoas conseguem adaptar-se com maior facilidade aos desafios de cuidar de alguém e experimentam um sentimento de apoio e proteção frente aos obstáculos (OLIVEIRA DA, et al., 2021).

A espiritualidade representa, uma dimensão universal e intrínseca do ser humano, uma vez que envolve significados, propósitos e valores humanos na busca de sentido para sua existência. Além disso, não necessita obrigatoriamente se relacionar de forma direta com uma instituição de caráter religioso (ROCHA RCNP, et al., 2018).

Assim esse artigo teve como objetivo analisar as condições que interferem na qualidade de vida dos cuidadores de pacientes oncológicos e a influência da espiritualidade nesse contexto.

\section{REVISÃO BIBLIOGRÁFICA}

A palavra cuidar se origina do latim e está relacionada ao ato de preservar, mostrar atitude de desvelo, zelar, demonstrar interesse e atenção. O cuidado expressa o sentimento de preocupação com o próximo e de inquietação pela pessoa estimada. Esta ação acontece em um momento de interação com o outro, quem cuida também se dispõe a entender o contexto e o mundo de quem recebe o cuidado, ajustando-se de acordo com as modificações que envolvem a totalidade do indivíduo (LEITE PS, et al., 2018).

O cuidado é uma característica própria do ser humano, visto como uma maneira de sobrevivência e de interligação de um ser com o outro e com o meio que o envolve. Essa ação de cuidar está relacionada com componentes cognitivos e comportamentais, se apresentando de forma multidimensional. O cuidar pode ser 
uma ação aprendida, que compreende o campo individual ou recíproco, quando prestamos a nós mesmos ou quando somos levados a prestar a outras pessoas que, provisoriamente ou permanentemente, tem necessidade de receber cuidados para a manutenção do estado vital (SILVA GS, et al., 2020).

Ao desempenhar o cuidado, o indivíduo se disponibiliza a atuar além da demanda física do paciente, de maneira a acolher e entender as particularidades, limitações e necessidades do outro, fornecendo inclusive, carinho, atenção e empatia. Essa atuação abrange questões emocionais, história de vida e princípios, de ambos os lados. Ademais, o cuidador informal é o sujeito, que fornece apoio direcionado à outra pessoa, que esteja necessitando de auxílio nas suas atividades diárias, podendo ou não possuir alguma ligação familiar com este. São vários os fatores que motivam a decisão para ser cuidador como a ligação familiar ou intensidade da relação, estar mais próximo do ambiente em que se encontra o paciente, a falta de outra alternativa e auto delegação (SILVA CV e GASPODINI IB, 2021).

Quando se coloca uma pessoa responsável pela atenção ao enfermo, esse indivíduo é visto como um recurso a serviço do adoecido. Assumindo diversos papéis, acompanhando o paciente cotidianamente, representando um instrumento essencial no decurso do tratamento e reabilitação. O cuidador se torna uma espécie de instituição responsável por monitorar o estado de saúde do paciente, definir providências a serem tomadas nos casos de queixas e sinais de mal-estar, avaliar e acompanhar incessantemente a condição clínica do enfermo. Além disso, também se envolve afetivamente, criando relações, conexões e internalizando valores (SALAZAR-BARAJAS ME, et al., 2019).

Ser cuidador propicia experiências e sentimentos positivos como: zelo, gratificação, carinho, crescimento pessoal e espiritual, mesmo que a função afete vários níveis da vida; devido às diversas exigências e a grande demanda de necessidades decorrentes deste cuidado. Comumente, os membros da família que deveriam participar dos afazeres, entendem o cuidador, como se fosse um sacerdote ortodoxo, estes familiares agem de acordo com tais pensamentos, ao depositar todos os anseios e responsabilidades dos demais, no cuidador, que, muitas vezes, tem que explicar e dar satisfação do ato de cuidar. Em virtude disso, a culpa da família como um todo, em relação ao doente, é mascarada (VALE JMM, et al., 2019).

Ao tomar para si todo o cuidado de forma individual, o cuidador principal sente-se abandonado, pela falta de suporte e cooperação dos demais membros, que muitas vezes, não valoriza o seu serviço, e ainda, critica aquilo que ele faz. Desse modo, o cuidador desenvolve sentimentos negativos, de sofrimento, pois desempenha um papel que deveria ser cumprido por todos os componentes da família (GARCIA AS, et al., 2021).

Ao mesmo tempo em que se sente satisfeito por cuidar do enfermo e colaborar consideravelmente na sua melhora e no sucesso da terapêutica. Também sente-se exausto pela função, em consequência das transformações vividas, associada a falta de preparo e ausência de outros suportes no ato de cuidar (SALAZAR-BARAJAS ME, et al., 2019).

O indivíduo que exerce o papel de cuidado, acaba perdendo sua própria identidade, a família, o vê como o único responsável e com disponibilidade infinita e tolerância permanente, como se não tivesse vontade própria ou livre arbítrio, sua identidade é encoberta pelo papel de cuidador. Logo, é comum, este, afligir-se com a pressão por parte da família e se sentir só, com medo, triste pelo familiar doente, cheio de dúvidas e incertezas, tentando fazer o seu melhor (VALE JMM, et al., 2019).

Vivenciar o desconforto físico e emocional, e manter a dedicação constante direcionada a pessoa com câncer representa alguns dos pontos que cooperam para reduzir o bem-estar do cuidador (EUGENIO APL e SAVARIS LE, 2021). O autor do cuidado se aproxima cada vez mais do paciente, devido a afetividade despertada nas relações cotidianas, colocando em muitas situações a sua própria vida em segundo plano. Geralmente suas atitudes e foco passam a ser exclusivamente para o paciente debilitado (SILVA RKN, et al., 2018).

Entende-se o autocuidado como uma função regulatória, que possibilita às pessoas realizarem sozinhas atividades direcionadas à preservação da vida, saúde, desenvolvimento e bem-estar. Muitas vezes o cuidador, abandona seu próprio cuidado para voltar sua atenção apenas ao familiar adoecido. Esse fato é 
justificado pela divisão das responsabilidades do cuidado, que acaba sendo assumida principalmente pelo cuidador, quando outro membro da família se faz presente apenas de forma fragmentada e esporádica (VALE JMM, et al., 2019).

Diante dessa vivência, o cuidador pode se sentir sobrecarregado. Apresentando-se por vezes, mais vulnerável à falta de apoio social e exposto a fatores que induzem o esgotamento físico, emocional, mental e alterações negativas nas condições de sua qualidade de vida (MILLANI APO, et al., 2021). Além disso, o câncer não afeta apenas a saúde do paciente, mas também interfere na saúde do cuidador, que acaba abstendo-se dos cuidados pessoais, das práticas de lazer e recreação, entre outras diversas atividades diárias (FERREIRA MLSM, et al., 2018).

A sobrecarga é compreendida através de duas variáveis: subjetiva e objetiva. A perspectiva subjetiva refere-se a sobrecarga, derivada das reações, emoções e comportamentos do cuidador diante do cuidado, ligada à experiência vivida. Por outro lado, a forma objetiva corresponde com a apresentação relacionada às atividades direcionadas ao cuidado, do tempo conferido na sua execução, e das mudanças sofridas no contexto de vida e rotina do cuidador (SILVA GS, et al., 2020).

A sobrecarga de cada cuidador pode variar, de acordo com o estágio da doença ou com os aspectos ligados ao estado do paciente. Podendo passar por transformações negativas nas relações, isolamento social, ausência de lazer, até mesmo alterações em hábitos alimentares e comprometimento das dimensões básicas do ser humano (EUGENIO APL e SAVARIS LE, 2021).

A ação de cuidar de um paciente em fase terminal da doença é estressante e pode apresentar-se como fator de risco para a saúde do cuidador. Repercutindo na vida deste, através de sintomas de depressão, ansiedade, stress, tensão, diminuição da qualidade de vida, perturbação do sono, deterioração do funcionamento social e ocupacional, se transformando em um fardo (DELALIBERA M, et al., 2018).

$\mathrm{Na}$ área da oncologia, o foco de atenção, na atuação dos profissionais de saúde, na maioria das vezes, também prevalece sobre o indivíduo doente, cabendo ao cuidador uma posição mais à margem dos acontecimentos. Ainda hoje, os cuidadores familiares são conhecidos como um elemento de benefício ao outro, mas não como objeto de atenção da equipe de saúde (MIGUEL I, et al., 2019). Em razão disso, o compromisso de cuidar tem sido abordado como um significante elemento que afeta as capacidades funcionais, adicionado ao caráter crônico e as longas horas de atuação integral, correlaciona-se com repercussões negativas do cuidar, provocando alterações em ambos os aspectos da vida de quem cuida (ZORTÉA J, et al., 2018).

Além do mais, ao lidar com uma situação de doença oncológica, o cuidador reconhece a finitude da vida, essa perspectiva em relação ao câncer e a morte, gera a sensação de impotência diante da doença. Junto a isso, muitos dos cuidadores, vivenciam a insegurança, medo e culpa, por se sentirem despreparados para realizar as tarefas e os cuidados, devido à falta de informação e inexperiência com o ofício (SILVA RKN, et al., 2018).

Ao receber o diagnóstico de câncer, tanto a pessoa, como sua família e seus vínculos sociais sofrem com a circunstância e podem apresentar um comportamento psicológico de antecipação da dor da perda iminente, chamado de luto antecipatório, caracterizado por ser um curso que se desenrola quando a pessoa doente está viva. Há várias formas de viver esse luto: o luto social e espiritual devido a compreensão da finitude, o luto pessoal de cada sujeito, o luto de uma morte que sobrevém no futuro e o luto pela mudança da rotina e contexto familiar (SILVA GS, et al., 2020).

Quando surge algo que recorda a memória, sobre a natureza limitada e finita do ser humano, o confronto com o tempo, acusa a maior fragilidade de uma pessoa. Em frente a essas indagações, o cuidador se depara com um processo inevitável, que promove sensações angustiantes (SILVA RKN, et al., 2018). Em vista disso, muitas vezes, o cuidador, além de antecipar o luto do seu parente, para tentar suportar e superar tal perda e sofrimento, também, procura prestar seus serviços de forma mais satisfatória, reportando-se a Deus como fonte de aconchego, sustento e consolo espiritual, conferindo a doença um significado de transformação para conquistar uma vida melhor (FERREIRA MLSM, et al., 2018). 
Concentrando esforços para adaptar-se à situação, buscando meios de enfrentamento, para se ajustar no decorrer da patologia e no processo de reabilitação da pessoa cuidada. Assim, como meio de enfrentamento dos encargos do serviço, os cuidadores buscam estratégias. Sendo destacado o papel da religião como um relevante instrumento que a família comumente recorre. Constituindo-se em uma ferramenta que pode ser acessada como fonte de suporte e conforto, por ter a capacidade de proporcionar serenidade durante as contrariedades da doença (CUNHA AS, et al. 2018).

A espiritualidade está relacionada com a essência do ser humano, o fundamento da vida de cada pessoa, a força e a energia. Ela direciona os caminhos da vida, permitindo ao ser humano descobrir objetivos, força interior e significado para sua jornada. Através da espiritualidade muitas questões são resolvidas sobre a vida, seu propósito, e a conexão com o sagrado que pode ou não ser vivido no contexto de uma religião específica (VIANNA MLGS e SOUZA W, 2017).

As práticas diárias espirituais, auxiliam os cuidadores a enfrentar e aceitar a doença do familiar doente, além de permitir também uma postura mais positiva por parte do doente, ajudando na regulação de emoções negativas, proporcionando mais dignidade e uma melhor condição psicológica para desfrutar do momento presente e sentir-se mais útil. Por sua vez, o cuidador, olha a sua vida com outros olhos, como se fosse uma nova vida, perguntando-se sobre a oportunidade que é viver, construindo pensamentos benéficos, que cooperam positivamente na sua saúde e ajudam a encarar o cotidiano (RODRIGUES KM, et al., 2019).

O exercício da espiritualidade e as práticas religiosas são vistas pelos cuidadores como um recurso eficaz para o enfrentamento da sobrecarga decorrente do processo de cuidar dos pacientes. Normalmente, quando um membro adoece, todo seu núcleo familiar também sofre e sente os efeitos do momento vivenciado. Diante desse cenário, a espiritualidade pode servir enquanto recurso terapêutico nos casos de sofrimento psíquico após o diagnóstico de câncer no âmbito familiar. Além disso, pesquisas revelam o importante papel desse meio no controle efetivo do estresse, ajudando as pessoas a lidar com situações de incertezas (CARBOGIM FC, et al., 2019).

Ademais, analisando o processo de cuidar no contexto domiciliar, a partir dos diversos aspectos que envolvem a qualidade de vida e espiritualidade, compreendemos que o cuidador principal também pode vir a adoecer, sofrendo efeitos psicológicos, comportamentais e fisiológicos durante esse processo, os quais afetam sua vida e saúde. A manutenção da saúde do cuidador informal é essencial para a prática saudável do ofício. Quando ocorre o reconhecimento dos fatores que levam a vulnerabilidades e proteção do cuidador informal principal do paciente com câncer, há a possibilidade de melhora da qualidade de vida do mesmo (MENEZES RR, et al., 2018).

\section{CONSIDERAÇÕES FINAIS}

O suporte oferecido por profissionais capacitados, a facilidade de acesso aos meios de saúde, a produção de conhecimento sobre os diversos aspectos da doença, o autocuidado e a participação dos componentes da família na realização de tarefas, são algumas das condições que geram proteção ao cuidador. Esses fatores, associados também, a medidas e políticas voltadas para os cuidadores, tais como acompanhamento psicológico, orientação e o livre exercício da sua crença, podem reduzir o estresse, auxiliar na redução da sobrecarga e melhorar a qualidade de vida na trajetória da atenção prestada. Ao ressaltar o cuidador como elemento que necessita de atenção, podemos prevenir o seu adoecimento e garantir a continuidade da realização dos cuidados ao familiar dependente.

\section{REFERÊNCIAS}

1. ABREU AISCS, JÚNIOR ALC. Sobrecarga do cuidador familiar de paciente oncológico e a enfermagem. Revista de enfermagem UFPE online, 2018; 12(4): 976-986.

2. CARBOGIM FC, et al. Enfrentamento de familiares de pacientes em tratamento oncológico. Revista de enfermagem e atenção à saúde, 2019; 8(1):51- 60 .

3. COPPETTI LC, et al. Habilidade de cuidado e sobrecarga do cuidador familiar de pacientes em tratamento oncológico. Revista Texto e Contexto - Enfermagem, 2020; 29: e20180451. 
4. CUNHA AS, et al. Cuidado paliativo oncológico: percepção dos cuidadores. Journal of Health \& Biological Sciences, 2018; 6(4): 383-390.

5. DELALIBERA M, et al. Circunstâncias e consequências do cuidar: caracterização do cuidador familiar em cuidados paliativos. Ciência \& saúde coletiva, 2018; 23(4): 1105-1117.

6. EUGENIO APL, SAVARIS LE. A vivência de cuidadores/as de doentes de câncer: impactos, desafios e estratégias de enfrentamento. Revista de Saúde Pública do Paraná, 2021; 4(3): 115-131.

7. FERREIRA MLSM, et al. Ser cuidador de familiar com câncer. Ciencia y enfermería. Concepción, 2018; 24(6): 1-11.

8. GARCIA SA, et al. Comprometimento da qualidade de vida dos cuidadores de pacientes em tratamento paliativo. Revista Eletrônica Acervo Científico, 2021; 19: e6046-e6046.

9. LEITE PS, et al. Qualidade de vida dos cuidadores familiares: uma revisão integrativa. Revista Eletrônica Acervo Saúde, 2018; 15: 1914-1920.

10. MENEZES RR, et al. Qualidade de vida relacionada à saúde e espiritualidade em pessoas com câncer. Revista Brasileira de Cancerologia, 2018; 64(1): 9-17.

11. MIGUEL I, et al. Qualidade de vida e sobrecarga do cuidador informal principal do doente oncológico em diferentes fases de tratamento. Revista Portuguesa de Oncologia, 2019; 3(1): 13-20.

12. MILLANI APO, et al. Sobrecarga de los cuidadores familiares de pacientes en tratamiento oncológico. Cultura de los Cuidados; 2021; 25(60): 290-304.

13. OLIVEIRA DA, et al. O Cuidar de uma Pessoa com Câncer na Ótica de Familiares Cuidadores: Revisão de Literatura. Revista Espaço Ciência e Saúde, 2021; 9(2): 53-69.

14. OLIVEIRA MBP, et al. Atendimento domiciliar oncológico: percepção de familiares/cuidadores sobre cuidados paliativos. Escola Anna Nery Revista de Enfermagem, 2017; 21(2): e20170030.

15. ROCHA RCNP, et al. Necessidades espirituais vivenciadas pelo cuidador familiar de paciente em atenção paliativa oncológica. Revista Brasileira de Enfermagem, 2018; 71: 2635-2642.

16. RODRIGUES KM, et al. Cuidados paliativos e espiritualidade no câncer: um estudo bibliométrico. Nursing, 2019; 22(258): 3302-3306.

17. SALAZAR-BARAJAS ME, et al. Funcionamento familiar, sobrecarga e qualidade de vida no cuidador do idoso com dependência funcional. Enfermería universitaria, 2019; 16(4): 362-373.

18. SANTANA ACO, et al. Mídias digitais e cuidadores da pessoa com câncer: comunicação em saúde e apoio psicossocial. Revista Família, Ciclos de Vida e Saúde no Contexto Social, 2021; 9(1): 141-150.

19. SILVA BCA, et al. Vivências de familiares de pacientes com câncer: revisitando a literatura. Revista da SPAGESP, 2019; 20(1): 140-153.

20. SILVA CV, GASPODINI IB. A influência da participação familiar no tratamento do paciente oncológico. Revista Ciência e Humanização do Hospital de Clínicas de Passo Fundo, 2021; 1(1): 74-88.

21. SILVA GS, et al. O apoio familiar no tratamento do paciente oncológico: uma revisão narrativa. Revista da Saúde da AJES, 2020; 6(12): 46-58.

22. SILVA RKN, et al. Nível de estresse dos cuidadores de pacientes com câncer em fase terminal. Revista Expressão Católica Saúde, 2018; 2(2): 53-61.

23. TAVARES DS, et al. Perfil epidemiológico de pacientes oncológicos em um serviço secundarista. Revista Brasileira de Educação e Saúde, 2020; 10(1): 122-128.

24. VALE JMM, et al. Autocuidado do cuidador de adoecidos em cuidados paliativos oncológicos domiciliares. Revista de enfermagem UFPE online, 2019; 13: 1-11.

25. VIANNA MLGS, SOUZA W. A espiritualidade dos cuidadores informais de pacientes em cuidados paliativos: uma reflexão bioética na perspectiva da alteridade. Estudos Teológicos, 2017; 57(2): 401-413.

26. ZORTÉA J, et al. Perfil nutricional e qualidade de vida de cuidadores de crianças e adolescentes com câncer. Clinical and biomedical Research, 2018; 38(1): 74-80. 